\title{
Can the first radiofrequency application for ablation of atrial tachycardia be successful in a patient after Fontan correction? With a little bit of luck... yes
}

\author{
Michał Orczykowski ${ }^{1}$, Piotr Zieliński², Marcin Demkow³ ${ }^{3}$ Andrzej Hasiec ${ }^{1}$, Maciej Sterliński' , Piotr Hoffman², \\ Łukasz Szumowski ${ }^{1}$
}

$11^{\text {st }}$ Department of Arrhythmia, National Institute of Cardiology, Warszawa, Poland 2Department of Congenital Heart Diseases, National Institute of Cardiology, Warszawa, Poland

${ }^{3}$ Department of Coronary and Structural Heart Diseases, National Institute of Cardiology, Warszawa, Poland

Correspondence to: Michał Orczykowski, MD, PhD, $1^{\text {st }}$ Department of Arrhythmia, National Institute of Cardiology, Alpejska 42, 04-628 Warszawa, Poland,

phone: +48696775533, e-mail:mikeorczyk@gmail.com

Copyright by the Author(s), 2022 DOI: 10.33963/KP.a2021.0140

Received:

September 27, 2021

Accepted:

October 18, 2021

Early publication date: October 19, 2021
A 42-year-old woman with a history of a single ventricle secondary to tricuspid valve atresia, who underwent a left Blalock-Taussig shunt at the age of 2, a Fontan atrio-pulmonary connection at the age of 10 , radiofrequency ablation of atrial tachycardia (AT) at the age of 28 , a conversion to a Glenn connection with a $22 \mathrm{~mm}$ collagen-coated woven polyester intra-atrial tunnel, partial removal of the interatrial septum, and surgical ablation with epicardial pacemaker implantation at the age of 36, was admitted to our institute due to an incessant AT and worsening symptoms of heart failure.

After careful evaluation of the clinical data, due to resistance to pharmacotherapy AT, we decided to perform radiofrequency catheter ablation. The procedure was performed under general anesthesia. We inserted 2 sheaths into the right femoral vein. From the intra-atrial tunnel, we recorded the AT, which had a cycle length of $350 \mathrm{~ms}$.

Under transesophageal echocardiography guidance (Figure 1A) and with computed tomography visualization of the intra-atrial tunnel course (Figure 1B), we were able to puncture the intra-atrial tunnel (Figure 1C, D) and successfully dilate the hole in the punctured polyester material.

We attempted to use an angioplasty balloon, but after the first contrast injection, it was discovered that the balloon leaked and during subsequent maneuvers, the guidewire was displaced. Successful insertion of the long sheath was achieved with the use of a typical, transseptal-like approach - a needle and a long sheath.

We performed a brief 3-dimensional activation map of the right atrium (Figure 1E) with the use of the CARTO 3 system (Biosense Webster, Inc., Diamond Bar, CA, USA) and a reference located within the intra-atrial tunnel. We recorded the low voltage, fractionated potentials at the lower part of the atrium (Figure 1F), and with entrainment maneuvers, we confirmed the area as the part of arrhythmia activation. First radiofrequency energy delivery restored the sinus rhythm. We were able to eliminate all fragmented signals in the area and an aggressive programmed stimulation protocol failed to induce any arrhythmias.

Procedure and fluoroscopy times were 310 and 73 minutes, respectively, and radiation dose was $13683 \mathrm{cGy} \mathrm{cm}^{2}$ and mostly related to transtunnel puncture. The patient's oxygen saturation (98\%) was unchanged from her prior value before the transtunnel puncture. No complications after the procedure have been noted. The patient was discharged from the hospital with sinus rhythm and a significant clinical improvement. There were no recurrences of arrhythmia at her 6-month follow-up.

In children with univentricular hearts, the introduction of a Glenn shunt followed by the breakthrough correction described by Fontan and Baudet, and intracardiac and extracardiac total cavopulmonary connection techniques, have improved the outcomes and survival of affected patients. The last 2 techniques are as- 


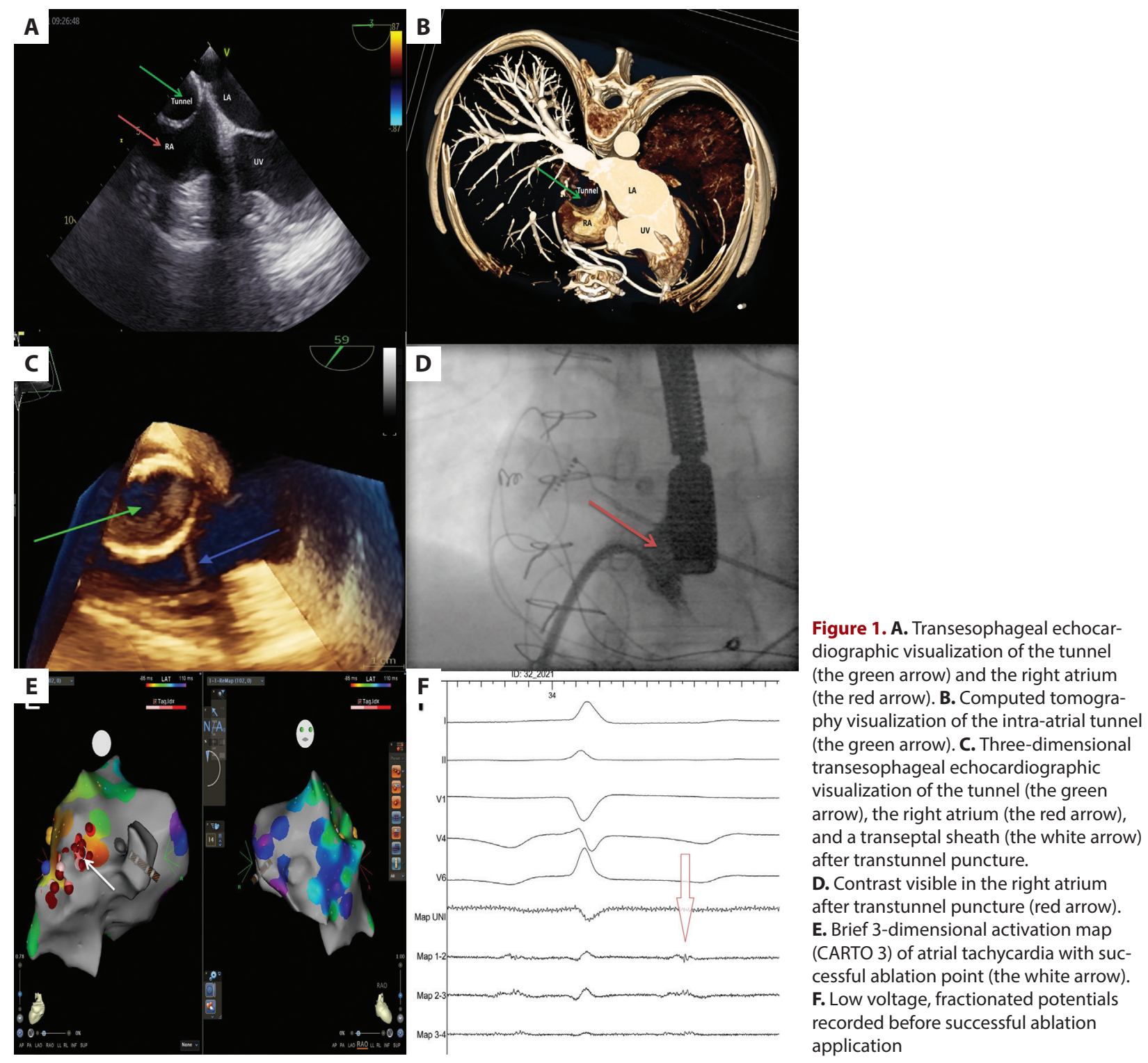

sociated with a lower risk of atrial arrhythmias [1]. However, cases in which an arrhythmia occurs can be a real challenge for electrophysiologists.

The implementation of the transtunnel (transbaffle) puncture technique (besides the remote navigation) provides an opportunity for successful ablation of the arrhythmia even in the presence of complex anatomy [2-5].

\section{Article information}

Conflict of interest: None declared.

Open access: This article is available in open access under Creative Common Attribution-Non-Commercial-No Derivatives 4.0 International (CC BY-NC-ND 4.0) license, allowing to download articles and share them with others as long as they credit the authors and the publisher, but without permission to change them in any way or use them commercially. For commercial use, please contact the journal office at kardiologiapolska@ptkardio.pl.

\section{REFERENCES}

1. Derejko P, Rybicka J, Biernacka EK, et al. Atrial tachycardia ablation in patients with a functional single ventricle after the Fontan surgery. Kardiol Pol. 2016; 74(8): 762-771, doi: 10.5603/KP.a2015.0214, indexed in Pubmed: 26575308.

2. Nehgme RA, Carboni MP, Care J, et al. Transthoracic percutaneous access for electroanatomic mapping and catheter ablation of atrial tachycardia in patients with a lateral tunnel Fontan. Heart Rhythm. 2006; 3(1):37-43, doi: 10.1016/j.hrthm.2005.09.027, indexed in Pubmed: 16399050.

3. Ernst S, Babu-Narayan SV, Keegan J, et al. Remote-controlled magnetic navigation and ablation with $3 \mathrm{D}$ image integration as an alternative approach in patients with intra-atrial baffle anatomy. Circ Arrhythm Electrophysiol. 2012; 5(1): 131-139, doi: 10.1161/CIRCEP.111.962993, indexed in Pubmed: 22062797.

4. Baszko A, Czyż K, Surmacz R, et al. Transbaffle radiofrequency ablation of reentrant atrial tachycardia in a child with hypoplastic left heart syndrome after Fontan correction. Kardiol Pol. 2015; 73(7): 572, doi: 10.5603/KP.2015.0128, indexed in Pubmed: 26189474.

5. Gałeczka M, Kowalski O, Fiszer R. Fontan tunnel puncture with 3-dimensional image fusion guidance for ablation of supraventricular arrhythmia in a patient with unique anatomy. Kardiol Pol. 2021;79(7-8): 873-874, doi: 10.33963/kp.15971, indexed in Pubmed: 33909387. 